\title{
Recruitment Threshold and Muscle Fiber Conduction Velocity of Single Motor Units
}

\author{
Tadashi Masuda and ${ }^{*}$ Carlo J. De Luca \\ Industrial Products Research Institute, Tsukuba, Ibaraki, Japan; and *NeuroMuscular Research Center, \\ Boston University, Boston, Massachusetts, U.S.A.
}

\begin{abstract}
Summary: The average muscle fiber conduction velocity (CV) measured with multicontact surface electrodes has been reported to increase with the contraction force. To understand this behavior better, we studied the relationship between the recruitment threshold and the muscle fiber $\mathrm{CV}$ of single motor units (MUs). Myoelectric signals were recorded simultaneously with a linear surface electrode array and a selective needle electrode. From the signals detected by the needle electrode, the discharges of single MUs were isolated by the decomposition technique. By using the firing of single MUs as the trigger point, we averaged the surface myoelectric signals and extracted the single MU action potentials from the interference surface signals. The $\mathrm{CV}$ of single MUs, calculated by a cross-correlation analysis, was higher for MUs recruited at higher contraction force. This result indicates that the larger MUs with higher muscle fiber CV contribute to increase the average CV during varying force contractions. Key Words: Single motor unit-Decomposition-Recruitment threshold-Muscle fiber conduction velocity-Tibialis anterior.
\end{abstract}

Development of the surface electrode array technique has made it possible to measure the muscle fiber conduction velocity (CV) noninvasively from the skin surface during a voluntary contraction of up to the maximal force $(6,9)$. With this technique, many investigators have reported that the average muscle fiber $\mathrm{CV}$ increases with the contraction force $(2,3,13)$. One of the possible reasons for this increase is that motor units (MUs) with higher recruitment thresholds have muscle fibers with larger diameters and consequently have higher muscle fiber $\mathrm{CV}$. Another factor affects the $\mathrm{CV}$, however, i.e., the firing rate of the MUs $(11,14)$.

Accepted December 11, 1990

Address correspondence and reprint requests to Dr. T. Masuda at Industrial Products Research Institute, Tsukuba, Ibaraki 305, Japan.
By electrically stimulating single motor nerve fibers, Andreassen and Arendt-Nielsen (1) measured the muscle fiber $\mathrm{CV}$ of single MUs and compared it with the size of the MUs, which was estimated from the response of the twitch torque. They reported the CV to be greater for larger MUs and called this association the size principle of the $\mathrm{CV}$. They did not study the CV during a voluntary contraction, however, in which the firing rate affects the CV.

Sadoyama and Masuda (12) measured the CV of single MU action potentials (MUAPs) and calculated the average $\mathrm{CV}$ from the interference surface myoelectric (ME) signals. They showed that the increase of the average $\mathrm{CV}$ is partially due to the firing rate of MUs, but the change of the $\mathrm{CV}$ caused by the firing rate was less than the change in the average $\mathrm{CV}$. Apparently the change of the $\mathrm{CV}$ caused by the increase of firing rate alone was insufficient to explain the change of the average $\mathrm{CV}$. 
If two parameters are assumed to be linearly related, the MUs with higher recruitment threshold will have a higher $\mathrm{CV}$ than the lower threshold MUs. However, the study of Sadoyama and Masuda (12) was limited to a few MUAPs isolated from the surface signals by a visual analysis, and the analysis was made only below $40 \%$ of the maximal voluntary contraction (MVC).

We attempted to reduce these limitations in the present study by studying MUs recruited up to $\sim 60 \%$ MVC. An ME signal decomposition technique (known as Precision Decomposition) developed by De Luca et al. $(7,15)$ was used to identify and categorize individual MUAPs from the ME signal detected with a specialized quadrifilar needle electrode. The surface ME signal was detected simultaneously, and the surface potentials of single MUs were extracted by trigger-averaging the surface interference ME signals with the firings of single MUs decomposed from the needle signals. We then calculated the CV of single MUAPs and compared it with the recruitment threshold of the individual MUs.

\section{METHODS}

The subjects were 3 healthy normal adults ( 2 men and 1 woman) aged 24, 24, and 35 years. All signed an informed consent document before participating in the experiment. The ME signals were recorded from the tibialis anterior. This muscle was used because the needle signals are relatively stable during a varying force contraction as compared with other muscles, and the muscle fibers are long and arranged in parallel fashion. Such architecture is conducive to detecting propagation of the MUAPs with the surface electrode array.

The recording procedure was described in a separate paper (8). The surface electrode array consisted of 17 silver contacts $10.0 \mathrm{~mm}$ wide and 1.0 $\mathrm{mm}$ thick arranged parallel to each other. The pitch of the contacts was $5.08 \mathrm{~mm}$. Sixteen signals were derived bipolarly from pairs of adjacent contacts with a gain of 2,000 and were digitized at a rate of 5 $\mathrm{kHz}$. The surface electrode was placed on the distal portion of the tibialis anterior, and the potentials arising from the most distal innervation zones and propagating in the distal direction were analyzed. The most distal innervation zone is located near one third of the distal length of the tibialis anterior (10). In the proximal region of this muscle, propagation of MUAPs is not detectable with the surface elec- trode array, probably because the muscle fibers are arranged in a more complex configuration.

The selective needle electrode had four contacts at the side of the cannula. We derived three signals between the contacts or between the contact and the cannula. The needle was inserted at the proximal edge of the surface electrode into the distal direction. The position of the needle electrode was adjusted to yield spikes of single MUAPs for the decomposition analysis. The needle signals were digitized at $50 \mathrm{kHz}$. The signal was compressed when it contained no APs exceeding the predetermined threshold, typically $\sim 1 \mathrm{mV}$. The sampled signals were then decomposed into trains of single MUAPs with the aid of the computer algorithm called Precision Decomposition $(7,15)$. The discharges of the isolated MUAPs were used as the trigger point for averaging the interference surface ME signals.

In addition to the ME signals, we measured the contraction force of dorsiflexion around the ankle. The force level was displayed to the subject, who produced the indicated force trajectory by watching a monitor screen. The force trajectory was trapezoidal. The subject reached the indicated force in 10 $\mathrm{s}$, sustained the force for $10 \mathrm{~s}$, and decreased the force to relaxation in 5-10 s. The target force level was $75 \% \mathrm{MVC}$. At contraction levels $>75 \% \mathrm{MVC}$, it is difficult to sustain the force for $10 \mathrm{~s}$, the time required to extract the surface MUAPs for single MUs by the trigger-averaging method.

The surface signals were averaged during the sustained contraction of $75 \%$ MVC because the CV and the firing rate may change during a period of increasing or decreasing force. The muscle fiber $\mathrm{CV}$ was calculated from the time shift between two signals recorded $10.16 \mathrm{~mm}$ (two channels) apart. The time shift was estimated by the cross-correlation analysis. The two signals used for the calculation were chosen from channels that yielded the highest cross-correlation. Besides the $\mathrm{CV}$ of single MUAPs, the CV of interference surface ME signals was calculated every $0.5 \mathrm{~s}$, also by cross-correlation analysis.

\section{RESULTS}

Figure 1 shows the discharges of seven single MUAPs decomposed from the needle ME signals. From the other two subjects, we isolated six and five MUs from a single record (Table 1). The discharges of MUAPs with small amplitude, e.g., MU 5 in Fig. 1, were not completely detected when other 


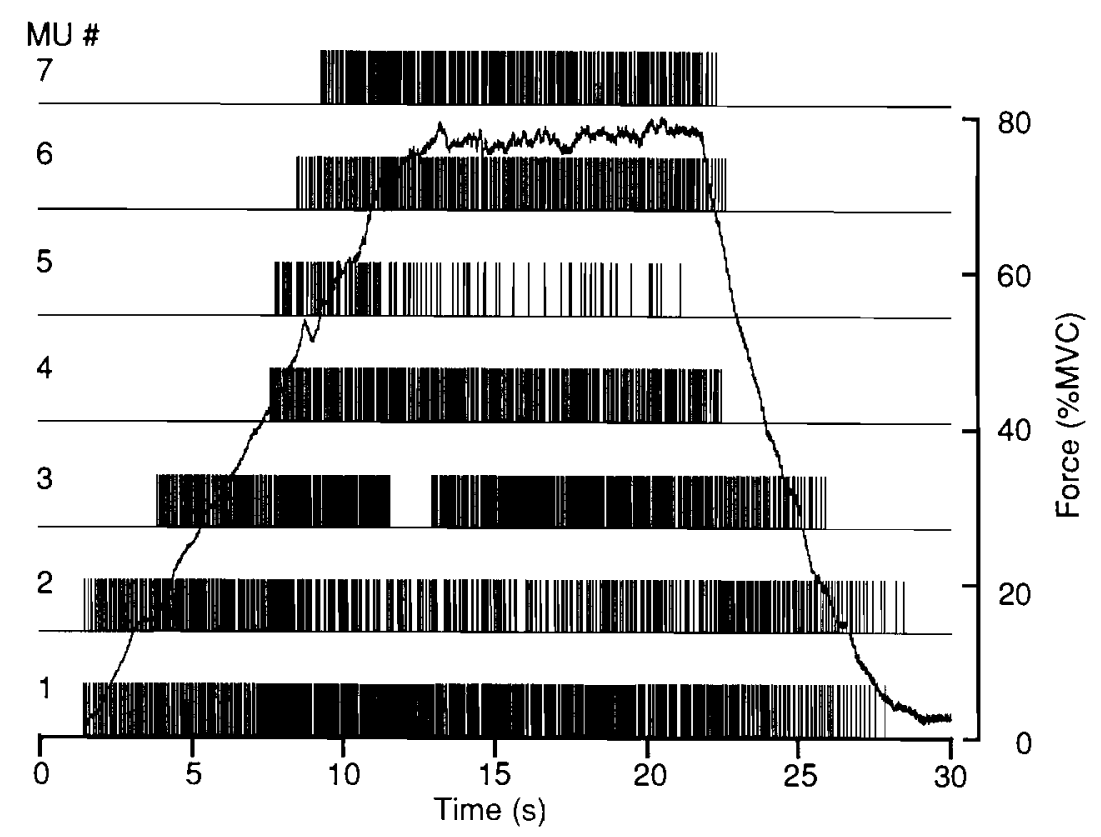

FIG. 1. Discharges of single motor units (MUs) decomposed from the needle myoelectric signals. Contraction force is also shown. Recruitment threshold was estimated from the force when the first discharge of MUs occurred. MUs 3 and 5 were not decomposed with complete accuracy (some action potentials were missed). This is not detrimental to the procedure used in this technique.

large-amplitude MUAPs were superimposed. The accuracy of the decomposition from the other two subjects was better than that for subject T.M. (Fig. 1 ). The accuracy is apparent in the number of firings used to trigger-average the surface ME signals, which was at least 187 and 147 for subjects N.P. and A.R., respectively. The mean interpulse interval was estimated for each MU based on its firing interval histogram.

Figure 2 shows the waveforms of the raw surface ME signals derived with the linear electrode array. There was a clear time shift from channel 7 to 13 in the distal direction and from channel 6 to 1 in the proximal direction. From this time shift, we calculated the average $\mathrm{CV}$. The source of the propagation located between channels 6 and 7 indicates the position of the innervation zones.

Figure $3 \mathrm{~A}-\mathrm{C}$ shows the average $\mathrm{CV}$ calculated every $0.5 \mathrm{~s}$ by the cross-correlation analysis. In all subjects, the $\mathrm{CV}$ increased with the contraction force. To calculate the amount of this increase, we defined the CV at low-level contraction as the average of $\mathrm{CV}$ values during a period of $<30 \% \mathrm{MVC}$ contractions. We also defined the $\mathrm{CV}$ at high-level contraction as the average of $\mathrm{CV}$ values during $75 \%$ MVC contractions. The average $\mathrm{CV}$ of the subject TM thus calculated increased from $3.1 \mathrm{~m} / \mathrm{s}$ (lowlevel contraction) to $4.1 \mathrm{~m} / \mathrm{s}$ (high-level contraction) (Fig. 3A). Similarly, the average CV of the other two subjects (N.P. and A.R.) increased from 3.2 to $3.9 \mathrm{~m} / \mathrm{s}$ and from 4.0 to $4.4 \mathrm{~m} / \mathrm{s}$, respectively. The ratio of increase in the average $\mathrm{CV}$ at $75 \% \mathrm{MVC}$ as compared with the $\mathrm{CV}$ at the low-level contraction $(<30 \%$ MVC) was therefore 31,23 , and $10 \%$ for subjects T.M., N.P., and A.R., respectively. The increase in $\mathrm{CV}$ averaged over the three subjects was $21 \%$. The increase in CV was not necessarily linear with the contraction force. In subject T.M., most of the increase in $\mathrm{CV}$ occurred around the force level of $40-55 \%$ MVC. This pattern of changes in the average $\mathrm{CV}$ was different between subjects. In subject N.P., the CV increased linearly over the range

TABLE 1. Parameters for averaging surface myoelectric signals and for calculating cross-correlation between two channels of averaged MUAPs

\begin{tabular}{|c|c|c|c|c|c|}
\hline \multirow[b]{2}{*}{ Subject } & \multirow[b]{2}{*}{ No. of MUs } & \multicolumn{2}{|c|}{ Average number } & \multicolumn{2}{|c|}{ Cross-correlation } \\
\hline & & Average & (Range) & Average & (Range) \\
\hline T.M. & 7 & 176.9 & ( $33-250)$ & 0.972 & $(0.949-0.986)$ \\
\hline N.P. & 6 & 230.3 & $(187-275)$ & 0.937 & $(0.877-0.979)$ \\
\hline A.R. & 5 & 205.4 & $(147-235)$ & 0.842 & $(0.671-0.989)$ \\
\hline
\end{tabular}




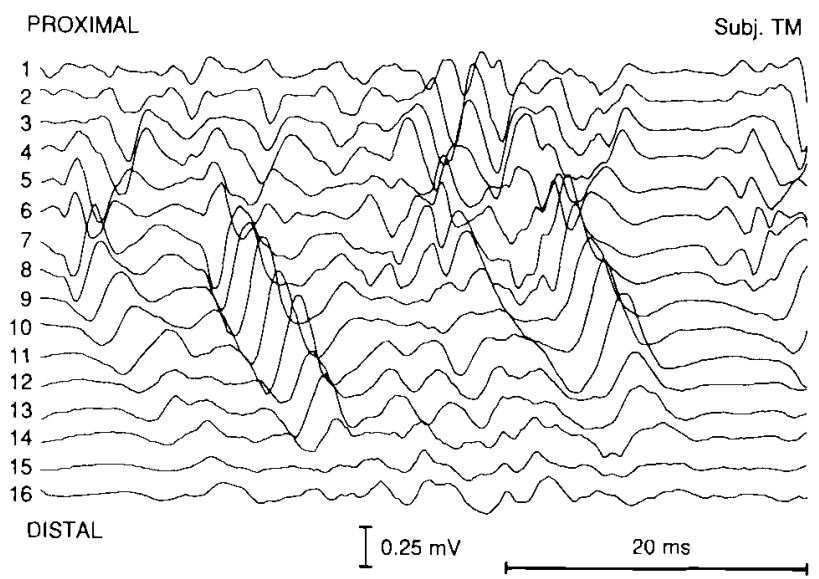

FIG. 2. A typical waveform of the surface myoelectric signals derived with a linear electrode array.

of $0-75 \%$ MVC, whereas in subject A.R. most of the increase in $\mathrm{CV}$ was observed in a force range $<50 \%$ MVC.

During the sustained contraction, the average $\mathrm{CV}$ decreased at a rate of $0.01-0.02 \mathrm{~m} / \mathrm{s}^{2}$, i.e., $0.1-0.2$ $\mathrm{m} / \mathrm{s}$ after the $10 \mathrm{~s}$ of $75 \% \mathrm{MVC}$ contraction. This decrease was the result of muscular fatigue, and because it was much smaller than the change in $\mathrm{CV}$ caused by the contraction level, we ignore the effect of muscular fatigue in the following analysis.

Figure 4 shows the trigger-averaged surface MUAPs of seven MUs in subject T.M. The averaging was performed during the constant force segment of the $75 \%$ MVC sustained contraction. The three lines in each trace indicate the average and the average $\pm 3 \mathrm{SD}$ and show the variability of the waveforms after the averaging. The MUs with smaller amplitude (MUs 1-3) and that with smaller number of averaging (MU 5) showed larger variability.

All 16 channels of the averaged MUAPs belonging to MU 4 are shown in Fig. 5. Clear propagation of MUAPs is evident in channels 7-11. The signals in channels 12-16 had smaller amplitude than those in channels 7-11. Some of the muscle fibers in this MU may have reached the tendon between channels 11 and 12 . The CV of this MU was $4.28 \mathrm{~m} / \mathrm{s}$, which was calculated by the cross-correlation between channels 9 and 11 . To clarify the effect of the firing rate and the muscle fatigue on the $\mathrm{CV}$ of single MUs, we tried to calculate the $\mathrm{CV}$ of individual MUs for a short period, e.g., $2 \mathrm{~s}$. Because of the smaller number of averagings, however, the CV estimated for this short interval was too variable and showed no clear tendency with time or firing rate.
Figure 6 shows the relationship between the recruitment threshold and the CV of individual MUs. Eighteen MUs detected from the three subjects are shown together. There was a significant correlation between the recruitment threshold and the $\mathrm{CV}$ of single MUs $(r=0.69, \mathrm{p}<0.01)$; i.e., the $\mathrm{CV}$ was greater for MUs with higher recruitment thresholds.

Figure 6 also shows that some MUs deviated from the regression line. This deviation may have been caused by the noise in the measurement. The seven MUAPs isolated from subject T.M. had a correlation coefficient of 0.97 on the average (Table 1) and fitted the regression line well, whereas the MUAPs from subjects N.P. and A.R. had correlation coefficients of 0.94 and 0.84 on the average, respectively. The MUs from these subjects showed a larger deviation (Fig. 6). These correlation coefficients are not small in the normal $\mathrm{CV}$ calculation, but because the difference of 1 sampling point $(0.2$ $\mathrm{ms}$ ) in the time shift causes a CV of $4 \mathrm{~m} / \mathrm{s}$ to be changed by $0.32 \mathrm{~m} / \mathrm{s}$ or $8 \%$, a record with these smaller correlation coefficients can show a larger variability in $\mathrm{CV}$ estimates.

Figure 7 shows the relationship between the recruitment threshold and the mean interpulse interval of individual MUs during the period of $75 \%$ MVC contractions. There was a significant tendency for the later recruited MUs to have longer interpulse intervals, i.e., lower firing rates $(r=$ $0.74, \mathrm{p}<0.01$ ). This observation is consistent with our previous work $(4,5)$. Because we know that an increase in the firing rate induces an increase in the $\mathrm{CV}$, we also know that the later recruited MUs generally have greater muscle fiber $\mathrm{CV}$ but generally lower firing rates than earlier recruited MUs. Therefore, the greater $\mathrm{CV}$ of the later recruited MUs is not strongly affected by their firing rates.

\section{DISCUSSION}

A statistically significant $(\mathrm{p}<0.01)$ correlation was noted between the recruitment threshold and the $\mathrm{CV}$ of individual MUs. This correlation indicates that the muscle fibers of MUs recruited at higher thresholds tend to have greater CVs. These data thus support the results of Andreassen and Arendt-Nielsen (1) obtained with the microstimulation technique. The method used by Andreassen and Arendt-Nielsen (1) has an advantage in that it can analyze the $\mathrm{CV}$ without being affected by the firing interval. Our study, on the other hand, provides one technical advantage: i.e., the set of 18 
3A,B

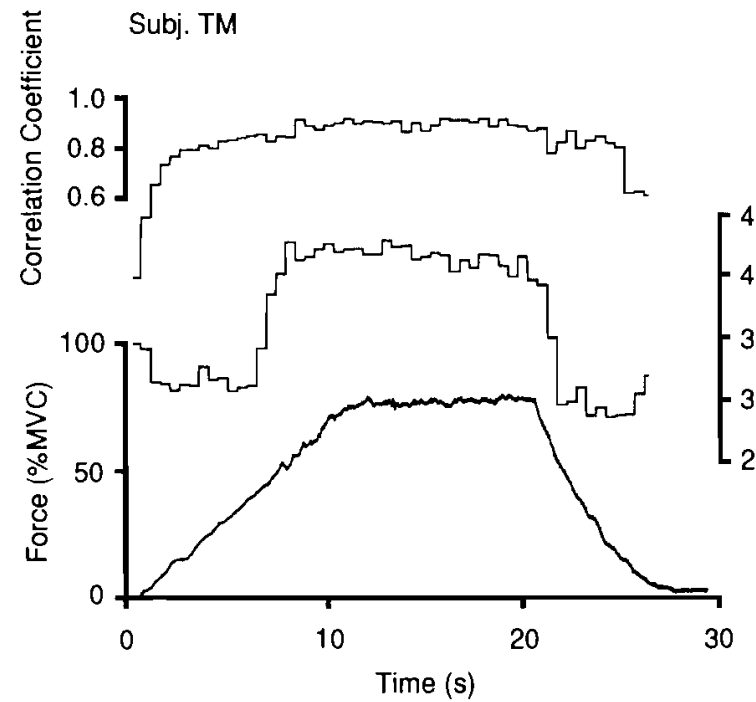

3C



MUs we studied were composed of different MUs, because by decomposing the ME signal, we obtain the APs of concurrently active MUs. This guarantee cannot be obtained in a study that uses electric stimulation to activate one MU at a time. This assurance of not measuring or not processing the data of a MU more than once has bearing on the correlation analysis.

Andreassen and Arendt-Nielsen (1) reported that prolonged and reliable tetanic stimulation of a single MU was difficult with the microstimulation technique. As a result of this difficulty, they could not assess whether the fatigue properties of the MUs were correlated to the size principle parameters. Although we have not tried this kind of experiment, the fatigue property can be clarified with the pres-



FIG. 3. Average muscle fiber conduction velocity (CV) calculated every $0.5 \mathrm{~s}$ from the interference surface myoelectric signals. $\mathrm{CV}$ was estimated from the time delay calculated by cross-correlation analysis. The correlation coefficient is also plotted for each 0.5-s period.

ent approach using the decomposition technique and should be a topic of further study.

The relationship between the size of MUs and the CV was suggested by Sadoyama and Masuda (12), who measured the $\mathrm{CV}$ of single MUs at their individual discharges and compared it with the recruitment threshold of MUs. They showed that the increase in CV within MUs is affected by the firing rate but is smaller than the increase of the average $\mathrm{CV}$ calculated over the full range of the contraction force. Based on this observation, they concluded that the increase in $\mathrm{CV}$ within MUs alone is insufficient to explain the increase of the average $\mathrm{CV}$. Their approach is advantageous because the CV and the firing interval can be measured at individual discharges of MUs. The same analysis cannot be 


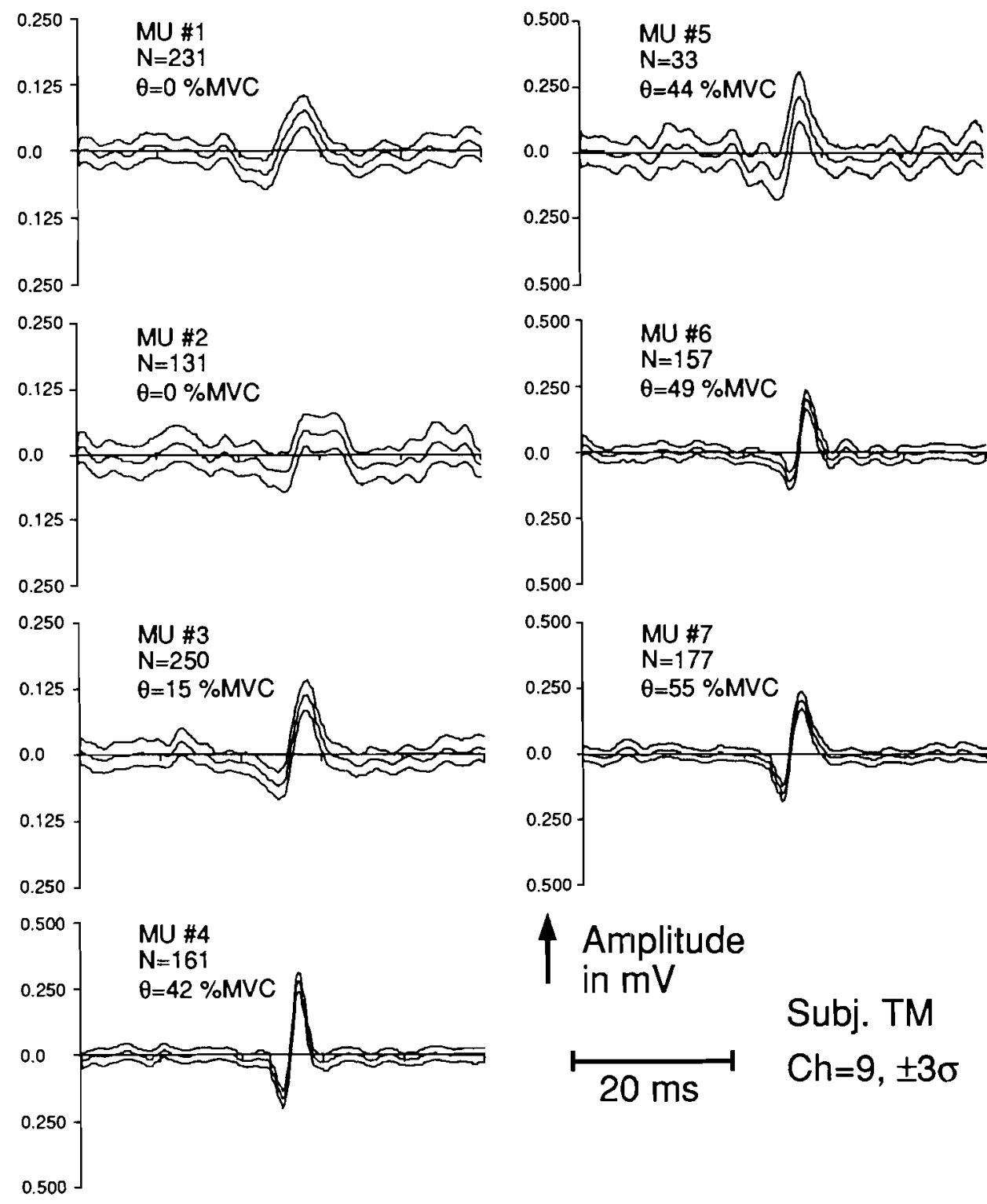

FIG. 4. Surface myoelectric signals trigger-averaged by the discharges of seven motor units shown in Fig. 1 for the period of $75 \%$ MVC sustained contraction. $N$, the number of averagings; $\theta$, recruitment threshold.

made with our method, because an averaging for a duration of $\sim 10 \mathrm{~s}$ is necessary to extract single MUAPs from the interference surface ME signals. Therefore, we cannot measure the change in $\mathrm{CV}$ within MUs or the dependence of $\mathrm{CV}$ on the firing interval.

In the method used by Sadoyama and Masuda (12), however, the ME signals were recorded only with a surface electrode and were analyzed only under the force level of $40 \% \mathrm{MVC}$; as a result of this limitation, they could not analyze high-threshold MUs and could not show any correlation between the recruitment threshold and the $\mathrm{CV}$ as we did.
In the present study, the amount of the increase of $\mathrm{CV}$ from low-level contraction ( $<30 \% \mathrm{MVC})$ to high-level contraction ( $75 \% \mathrm{MVC}$ ) was $21 \%$ on the average for the three subjects. Broman et al. (3), who measured the $\mathrm{CV}$ in the tibialis anterior of eight subjects, reported that the $\mathrm{CV}$ increased by an amount of $29 \%$ from $3.5 \mathrm{~m} / \mathrm{s}$ at $10 \%$ MVC to $4.5 \mathrm{~m} / \mathrm{s}$ at $100 \%$ MVC. Similarly, Arendt-Nielsen et al. (2), who measured the $\mathrm{CV}$ in the vastus lateralis of six subjects, reported that $\mathrm{CV}$ increased by $33 \%$ from $4.5 \mathrm{~m} / \mathrm{s}$ at $10 \%$ MVC to $6.0 \mathrm{~m} / \mathrm{s}$ at $100 \%$ MVC. Our observations indicate a range of increase similar to these reported values. 
PROXIMAL Subj. TM, MU \#4



FIG. 5. All 16 channels of the surface action potentials of motor unit (MU) 4 shown in Figs. 1 and 4. This MU had a conduction velocity of $4.28 \mathrm{~m} / \mathrm{s}$, which was calculated by the cross-correlation between the signals of channels 9 and 11 . The correlation coefficient was 0.98 for this record.

We wished to determine whether recruitment of MUs with higher $\mathrm{CV}$ is sufficient to explain the increase in average $\mathrm{CV}$ calculated from the interference surface $\mathrm{ME}$ signals recorded during a varying force contraction. The regression line between the recruitment threshold and the $\mathrm{CV}$ (Fig. 6) had an intersection of $3.45 \mathrm{~m} / \mathrm{s}$ at $0 \% \mathrm{MVC}$ and a gradient of $0.018 \mathrm{~m} / \mathrm{s} / \%$ MVC. This regression line indicates that when MUs with recruitment thresholds varying from 0 to $75 \%$ MVC become active the $\mathrm{CV}$ is ex-

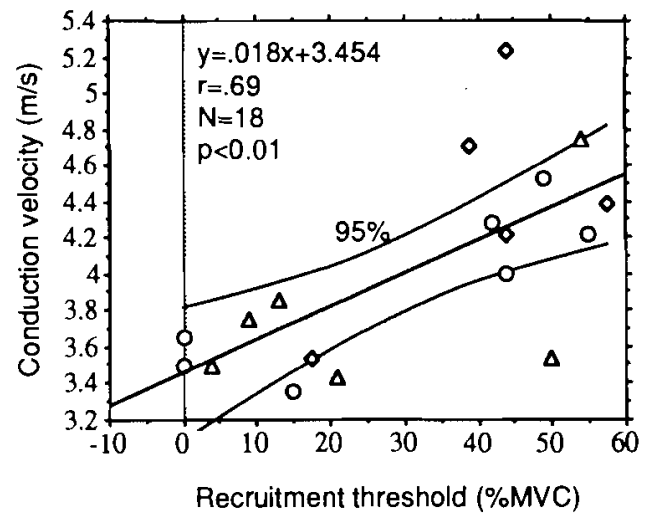

FIG. 6. Relationship between the recruitment threshold and the conduction velocity (CV) of 18 single motor units (MUs) obtained from three subjects: open circles, T.M.; open triangles, N.P.; open diamonds, A.R. The correlation between these quantities was statistically significant; i.e., the CV was higher for MUs with higher recruitment threshold.

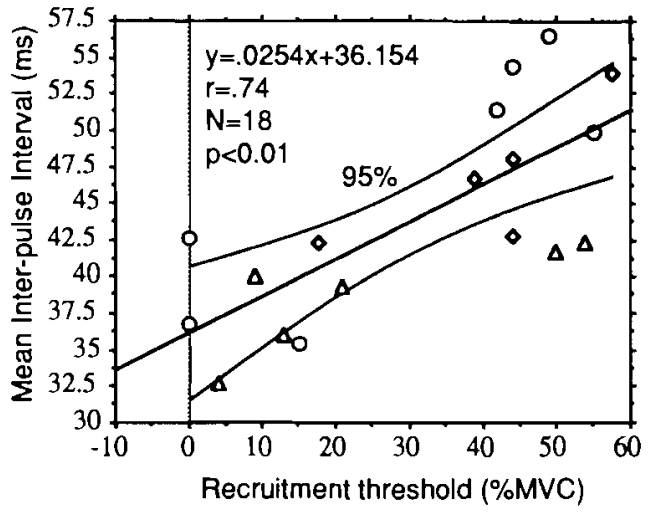

FIG. 7. Relationship between the recruitment threshold and the mean interpulse interval of motor units (MUs) during the $75 \%$ MVC sustained contraction. The later recruited MUs tended to have larger interpulse intervals, i.e., smaller firing rates. Subjects studied: open circles, T.M.; open triangles, N.P., open diamonds, A.R.

pected to change by an amount of $1.35 \mathrm{~m} / \mathrm{s}$-from 3.45 to $4.80 \mathrm{~m} / \mathrm{s}$. This amount corresponds to an increase of $39 \%$ as compared with the CV of 3.45 $\mathrm{m} / \mathrm{s}$ for MUs with the lowest recruitment threshold. Since both low and high recruitment threshold MUs are active at the high-level contraction of $75 \%$ $\mathrm{MVC}$, the expected average $\mathrm{CV}$, for all MUs active at $75 \% \mathrm{MVC}$, is about the middle value of 3.45 and $4.80 \mathrm{~m} / \mathrm{s}$. Thus, the expected CV is $4.13 \mathrm{~m} / \mathrm{s}$. This value is an increase of $20 \%$ from the lowest contraction. The actual increase in the average $\mathrm{CV}$ we observed was $21 \%$, which agrees well with our estimation. This agreement indicates that the increase in $\mathrm{CV}$ during a varying force contraction is mainly due to recruitment of MUs with higher CVs.

The increase in CV was not necessarily linear with contraction force. In subject T.M., CV increased mostly at the force level of $40-55 \%$ MVC. The other subjects showed different patterns. The similar nonlinear relationship between the average $\mathrm{CV}$ and the contraction force was also reported by Broman et al. (3). According to the conclusion in the previous paragraph that the increase in $\mathrm{CV}$ with contraction force is mainly caused by recruitment of MUs with higher CVs, the increase in the average $\mathrm{CV}$ at the force range of $40-55 \%$ in subject T.M. indicates that a substantial recruitment of MUs occurs at this force range. Because of this association, we may be able to estimate the recruitment of MUs by analyzing the $\mathrm{CV}$ estimated from the interference surface ME signals.

Acknowledgment: This research was performed at the NeuroMuscular Research Center, Boston University. We 
thank the staff of NeuroMuscular Research Center for assisting this study. The research was supported by the Liberty Mutual Insurance Company, U.S.A., and by the Science and Technology Agency, Japan.

\section{REFERENCES}

1. Andreassen S, Arendt-Nielsen L: Muscle fibre conduction velocity in motor units of the human anterior tibial muscle: A new size principle parameter. J Physiol 391:561-571, 1987.

2. Arendt-Nielsen L, Forster A, Mills KR: The relationship between muscle fibre conduction velocity and force in the human vastus lateralis. $J$ Physiol 353:6P, 1984.

3. Broman H, Bilotto G, De Luca CJ: Myoelectric signal conduction velocity and spectral parameters: influence of force and time, $A m J$ Physiol 58:1428-1437, 1985.

4. De Luca CJ, LeFever RS, McCue MP, Xenakis AP: Behavior of human motor units in different muscles during linearly varying contractions. J Physiol 329:113-128, 1982.

5. De Luca CJ, LeFever RS, McCue MP, Xenakis AP: Control scheme governing concurrently active human motor units during voluntary contractions. J Physiol 329:129-142, 1982.

6. Lynn PA: Direct on-line estimation of muscle fiber conduction velocity by surface electromyography. IEEE Trans Biomed Eng BME 26:564-571, 1979.

7. Mambrito B, De Luca CJ: A technique for the detection, decomposition and analysis of the EMG signal. Electroencephalogr Clin Neurophysiol 58:175-188, 1984

8. Masuda T, De Luca CJ: Technique for detecting MUAP propagation from high-threshold motor units. J Electromyogr Kinesiol 1:75-80, 1991.

9. Masuda T, Miyano H, Sadoyama $T$ : The measurement of muscle fiber conduction velocity using a gradient threshold zero-crossing method. IEEE Trans Biomed Eng BME 29:673-678, 1982.

10. Masuda T, Sadoyama T: Skeletal muscles from which the propogation of motor unit action potentials is detectable with a surface electrode array. Electroencephalogr Clin Neurophysiol 67:421-427, 1987.

11. Morimoto S, Masuda M: Dependence of conduction velocity on spike interval during voluntary muscular contraction in human motor units. Eur J Appl Physiol 53:191-195, 1984.

12. Sadoyama T, Masuda T: Changes of the average muscle fiber conduction velocity during a varying force contraction. Electroencephalogr Clin Neurophysiol 67:495-497, 1987.

13. Sadoyama T, Masuda T, Miyano H: Relationship between muscle fibre conduction velocity and frequency parameters of surface EMG during sustained contraction. Eur J Appl Physiol 51:247-256, 1983.

14. Stålberg E: Propagation velocity in human muscle fibers in situ. Acta Physiol Scand 70(suppl 278):1-112, 1986.

15. Stashuk D, De Luca CJ: Update on the decomposition and analysis of EMG signals. In Desmedt JE, ed: Computeraided electromyography and expert systems. Amsterdam, Elsevier Science Publishers, 1989, pp 39-47. 